\title{
Features of Orthodontic Treatment during Pregnancy
}

\section{Bayramova AN}

Obstetrician, Department of Gynecology, Russia

Corresponding author: Bayramova AN, Obstetrician, Department of Gynecology, Russia,

Received date: January 20, 2017; Accepted date: February 08, 2017; Published date: February 18, 2017

Copyright: @ 2017 Bayramova AN. This is an open-access article distributed under the terms of the Creative Commons Attribution License, which permits unrestricted use, distribution, and reproduction in any medium, provided the original author and source are credited.

Citation: Bayramova AN. Features of Orthodontic Treatment During Pregnancy. Crit Care Obst Gyne. 2017, 3:1.

\section{Abstract}

Pregnancy is a special period in a woman's life, which is associated with the restructuring of the body, due to gestation.

This period is a phase of active cultivation of a woman when she thinks about her appearance. Consequently, pregnancy often coincides with the phase of active orthodontic treatment in women of childbearing age, who make up a large percentage of the cohort of patients. From the above we can conclude pregnancy and orthodontic treatment often take place parallel to one another.

How justified is the purpose of orthodontic treatment during pregnancy? This is important both for the patient and for the doctor to answer today.

\section{Keywords: Pregnancy; Orthodonntic Treatment; Aligners}

\section{Introduction}

A pregnant woman during gestation should visit the dentist three times. Clean mouth is an important criterion for the health of women in general, and in particular the appointment of orthodontic treatment, as a necessary condition for it [1]. During pregnancy maintaining itself becomes more challenging due to the changes occurring in a woman's body.

Changes in the immune system, and hormonal fluctuations associated with pregnancy have an impact on the development of infections in the mouth in pregnant women [2]. Thus, it is proved that pregnant women with gum disease (gingivitis), increases the risk of premature birth and the likelihood of having a baby with low birth weight also increases.

During pregnancy in the mouth may occur the so called "pregnancy granuloma" or the "tumor pregnant." This is a single growth on the gum, like a tumor, which can change the set of teeth and bleeding [3]. The cause of education is often a lack of oral hygiene. Normally the build-up disappears after pregnancy, but in some cases it is removed surgically.

Tooth mobility is another disease of the oral cavity, which can occur during pregnancy and is often associated with gum disease, namely periodontitis - a disease of tissues surrounding the root of the tooth [4]. The researchers also report on the relationship of having periodontitis in pregnant women and the potential danger of early childbirth.

Physiological xerostomia (dry mouth) is a common complaint during pregnancy. Often it appears with some drugs as a side effect, such as antidepressants, antihistamines and others [5]. Xerostomia can also occur during sleep.

During pregnancy increases the number of patients with bruxism, in which there is uncontrolled simultaneous compression of both jaws, in which sound is reminiscent of gnashing. This is due to several factors, firstly, the pregnancy itself is a stress for the woman's body, while patients with unstable reactivity, which the body does not have the ability to quickly and firmly align the changes that have occurred in it as a result of internal and external stimuli, and after eliminating them quickly to return to its original state, are most susceptible to the appearance of this condition [6]. Also, due to physiological changes in the body of a woman is complicated to maintain oral health, which can in turn lead to diseases of the teeth, which can serve as one of the factors of bruxism appearance [7].

Changes in the composition of saliva during late pregnancy and during breast feeding may temporarily predispose to erosion and dental caries.

The foregoing is testified to the increasing need for rehabilitation of the oral cavity women before orthodontic treatment [8]. This should be considered as some of these manipulations can be contraindicated during pregnancy. In this regard, such holding best considered during planning pregnancy.

What are the main reasons for the possible negative impact of dental treatment in pregnant women?

Psycho emotional stress caused by the visit to the dentist, waiting for the pain, the presence of pain in dental disease and or treatment.

A horizontal position in the dental chair, especially in the later stages of pregnancy

Duration of the dental procedures. The use of drugs in the course of dental treatment (local anesthetics, tranquilizers, nonsteroidal anti-inflammatory drugs, systemic use of antibiotics, and others). 
In the treatment of essential features taking into consideration physiology during pregnancy, which we will discuss below.

In pregnant patients after 6 weeks of gestation modified type hyperdynamic circulation at that clinically manifest can increase heart rate and its content often reduced blood pressure (BP) in the P-trimester with increased pulse pressure, in the normal course of pregnancy can be heart rhythm disturbances in the form extrasystole BP may increase in II-m and III-trimester, as a manifestation of hypertension $2^{\text {nd }}$ half pregnancy [9]. Clinically, these changes in the dental administration may result in:

The emergence of cardio-respiratory failure due to an inadequate increase in cardiac output, heart rate, oxygen consumption and minute breathing volume in response to psycho-emotional and physical load horizontal position of the patient in a chair with a change in hemodynamic conditions and increased preload on the heart.

Increased risk of syncope fainting as a manifestation of symptomatic orthostatic collapse.

Occurrence of cardiac arrhythmias in the form extra systole usually do not require medical treatment.

Increased risk of hypertension of the second half of pregnancy until eklampsiy. Functioning of kidney during pregnancy is particularly sensitive to changes in body position. It is amplified in the position of pregnant and lying in the lateral position appearing urge to urinate.

When the term of $30-32$ weeks, $10 \%$ of women in a horizontal position on a rigid base, for example, in the dental chair, develops compression of the inferior vena cava syndrome [10]. Enlarged uterus, compressing the inferior vena cava, reduces blood flow to the heart, which leads to increased peripheral resistance, blood pressure drops and the deterioration of the utero-placental blood flow. Especially pronounced this syndrome in pregnancy complicated forms, and hypotension Increased intra-abdominal pressure, coupled with relaxation of smooth muscles of the gastrointestinal tract leads to the appearance and aggravation of gastroesophageal reflux disease, which is clinically manifested by heartburn, nausea, vomiting, sometimes pain in the sternum [11]. Horizontal position provokes the appearance of symptoms. Therefore, taking into account changes in the cardiovascular system, gastrointestinal tract and kidneys, in working with pregnant should be provided only in a semi-sitting position of the patient's dental chair Planning certain dental procedures in a pregnant woman, a dentist should be aware that the nature of reparative processes, remodeling of bone structures and jaws antibacterial protection differ significantly from the generally accepted norm. This is because, firstly, pregnancy develops negative iron balance, caused the greatest increase in demand due to the presence of the fetus and placenta and in nursing feeding in iron expenditure [12]. Secondly, during pregnancy develops calcium deficiency, especially during the first pregnancy, with no fatal significance in terms of osteopenia or osteoporosis in later, but clearly affects the state of hard tissue of teeth mother and remodeling processes in violation of the integrity of the bony structures of the jaw. And, finally, immune status of pregnant women and the number of different reduction functions of $T$ and B-lymphocytes [13]. Content and an increase in the activity of T-suppressors is necessary to suppress the immune response to alloantigens fetus. Moderate antigenic foreignness of the mother and the fetus is necessary for a controlled conflict between them, triggering placenta formation process. On the border of the mother and embryo tissues occur anaphylactic reactions leading to the formation micro thrombus shielding embryonic antigens from the mother's immune system [14]. The sensitivity of pregnant women to drugs is different from the sensitivity to them non pregnant women. Pregnant may develop anaphylactic shock and drug intoxication in those situations in which they may or may not occur in non-pregnant women [15]. According to Yoshimura concerning cases of pregnant death associated with the use of drugs should be considered that a pregnant woman is in before-shock state because physiological changes in hemodynamics and hormonal status, as well as increased metabolism during pregnancy may cause the development of her hypersensitivity to medicines. Thus, changes psycho physiological status of various types of homeostasis (the immune, hormonal, metabolic, metabolism of calcium and iron, etc.) with the development of the pregnancy and in the immediate postpartum period really makes a woman vulnerable to manifestations of the negative impact of any intervention including dental [16]. Dental treatment during pregnancy ontodontal for the purpose of treatment should be carried out in the second trimester of pregnancy or during pregnancy planning, which is preferable. By the beginning of the second trimester, the fetus is already formed systems and organs, so the probability of harm to him at times below. The first trimester and the last month of pregnancy is a contraindication to such. Another important issue that is facing an orthodontist before the treatment which research methods can be used in $b$ AC line? The first and most common method is x-ray study. Is it worth to use it when a pregnant patient or the pregnancy is a strong contraindication to the destination itself? In modern dentistry, such studies are carried out with the help of X-ray machine, which uses minimal radiation (exposure of only a few milliseconds), in addition to protect the fetus a woman puts on a special apron that It covers the chest, abdomen and pelvis. teleroentgenography (TWG). This method is used for determining the state of roots. This picture has a high resolution, which allows the specialist to see the full picture of the oral cavity. Orthopantomography (XCTD) [17]. It allows you to take a picture in front projection. The very picture is taken by a tomograph, which shows the state of the tooth roots and tissues. To maximize protection, X-ray examination should be performed in the second trimester, the first and last months of pregnancy is forbidden to do so, as in this period is the most vital organs of fetus [18]. During dental study strictly limited area is exposed to radiation any jaw side, the $\mathrm{X}$-rays are directed and do not dissipate. In addition, for added security, the body and the woman's abdomen protected by a lead apron, which does not transmit X-ray beams. The obvious advantage over X-ray survey of the during pregnancy has MRI, since this study completely excluded all types of $x$-ray. Contraindications MRI of the brain during pregnancy which are attributed by doctors are the first trimester of pregnancy, the presence of pacemakers, metal pins and needles in the body and implanted inhalers 
When there is evidence possibly holding ultrasound soft tissue of the face and neck, which in modern version does not require any special ultrasonic sensors or scanners and can be performed on the equipment intended for the study of peripheral structures: are ample linear sensors 5 oscillation frequency, 0-7, $5-9,0 \mathrm{MHz}$. The above features must be considered when preparing the pregnant patient to orthodontic treatment. At the same time the orthodontist must be guided by the recommendations of an obstetrician-gynecologist [19]. Subject to these conditions, guaranteed the success of the orthodontic treatment to achieve the desired results.

\section{Conclusion}

In this article we showed benefits of aligners during pregnancy comparing to braces.

\section{References}

1. Turner M, Aziz SR (2002) Management of the pregnant oral and maxillofacial surgery patient. J Oral Maxillofac Surg 60: 1479-1488.

2. Suresh R, Radfar L (2004) Pregnancy and lactation. Oral Surg Oral Med Oral Pathol and Oral Radiol Endod 6: 672-682.

3. Mabie, William C (1994) A longitudinal study of cardiac output in normal human pregnancy. Am J Obstet Gynecol 170: 849-856.

4. Lee W (1991) Cardiorespiratory alterations during normal pregnancy. Crit Care Clin 7: 763-775.

5. Hunter S, Robson SC (1992) Adaptation of the maternal heart in pregnancy. Br Heart J 99: 540-543.

6. Chu ZM, Beilin LJ (1993) Mechanisms of vasodilation in pregnancy: studies of the role of prostaglandins and nitric oxide in changes of vascular reactivity in the in situ blood perfused mesentery of pregnant rats. Br J Pharmacol 109: 322-329.

7. Theunissen IM, Parer JT (1994) Fluid and electrolytes in pregnancy. Clin Obstet Gynecol 37: 3-15.
8. Duvekot JJ, Peeters LL (1994) Maternal cardiovascular hemodynamic adaptation to pregnancy. Obstet Gynecol Surv 49: S1-S14.

9. Weiss G (2000) Endocrinology of parturition. J Clin Endocrinol Metab 85: 4421-4425.

10. Thornburg KL, Jacobson SL, Giraud GD, Morton MJ (2000) Hemodynamic changes in pregnancy. Semin Perinatol 24: 11-14.

11. Soory M (2000) Hormonal factors in periodontal disease. Dent Update 27: 380-383.

12. Tilakaratne A, Soory M, Ranasinghe AW, Corea SM, Ekanayake SL (2000) Periodontal disease status during pregnancy and 3 months post-partum in rural population of Sri-Lankan women. J Clin Periodontol 27: 787-792.

13. Yuan K, Wing LY, Lin MT (2002) Pathogenetic roles of angiogenic factors in pyogenic granulomas in pregnancy are modulated by female sex hormones. J Periodontol 73: 701-708.

14. Salvolini E, Di Giorgio R, Curatola A, Mazzanti L, Fratto G (1998) Biochemical modifications of human whole saliva induced by pregnancy. Br J Obstet Gynaec 105: 656-660.

15. Kauh YC, Zachian TF Melasma. Adv Exp Med Biol 455: 491-499.

16. Fiese R, Herzog $S$ (1988) Issues in dental and surgical management of the pregnant patient. Oral Surg Oral Med Oral Pathol 65: 292-297.

17. Rayburn WF (2002) Recommending medications during pregnancy: an evidence based approach. Clin Obstet Gynecol 45: 1-5.

18. Rathmell JP, Viscomi C, Viscomi MA (1997) Management of nonobstetric pain during pregnancy and lactation. Anesth Analg 85: 1074-1087.

19. FDA classification of drugs for teratogenic risk. (1994) Teratology 49: 446-447. 